\title{
Piezoelectric Materials Under Natural and Man-Made Radiation: The Potential for Direct Radiation Detection
}

\author{
Megan Wart, Evan Simpson, and Marek Flaska
}

\begin{abstract}
Radiation detection systems used for monitoring long term waste storage need to be compact, rugged, and have low or no power requirements. By using piezoelectric materials it may be possible to create a reliable self-powered radiation detection system. To determine the feasibility of this approach, the electrical signal response of the piezoelectric materials to radiation must be characterized. To do so, an experimental geometry has been designed and a neutron source has been chosen as described in this paper, which will be used to irradiate a uranium foil for producing fission fragments. These future experiments will be aimed at finding the threshold of exposure of lead zirconate titanate (PZT) plates needed to produce and electrical signal. Based on the proposed experimental geometry the thermal neutron beam-line at the Breazeale Reactor at The Pennsylvania State University will be used as the neutron source. The uranium foil and neutron source will be able to supply a maximum flux of $1.5 \mathrm{e} 5$ fission fragments $/ \mathrm{second}^{*} \mathrm{~cm}^{2}$ to each of the PZT plates.
\end{abstract}

Index Terms—radiation detection, fission fragments, lead zirconate titanate, piezoelectric materials

\section{INTRODUCTION}

$\mathrm{R}$ ADIATION detection is very important for maintaining the safety of nuclear facilities. Radiation detection systems are used to monitor radiation levels for personnel safety, as well as to ensure the integrity of nuclear materials storage, such as dry cask waste storage at reactor facilities, and interim or long term waste storage facilities. For monitoring waste storage it is useful to have a compact and rugged detection system that has low or no power requirements, since the waste containers are packed close together and are often exposed to the elements.

In an effort to create a self-powered detection system, the use of piezoelectric materials as a direct radiation sensing medium has been studied. Piezoelectric materials are able to convert between mechanical and electrical energy due to electric dipoles within the crystal structure of the material. When mechanically stressed, piezoelectric materials produce an electrical signal. Conversely, during the application of an electrical voltage the piezoelectric material will its change shape [1]. This behavior makes these materials useful for applications such as sensors, actuators, energy harvesting and generation, micro/nano-electronicmechanical systems, and piezoelectric igniters.

In previous work it has been shown that lead zirconate titanate (PZT) is sufficiently sensitive to produce an electrical signal when bombarded with high energy heavy ions. PZT samples were exposed directly to a $400 \mathrm{MeV} / \mathrm{n}$ xenon beam, resulting in a visible electrical signal [2]. It was also shown that the PZT samples could detect acoustic waves in liquid created by high energy xenon ions.

- M. Wart, E. Simpson and M. Flaska are with the Department of Mechanical and Nuclear Engineering, The Pennsylvania State University, State College, $P A, 16802$.

E-mail:mxf5309@psu.edu

Manuscript received July 15, 2017.

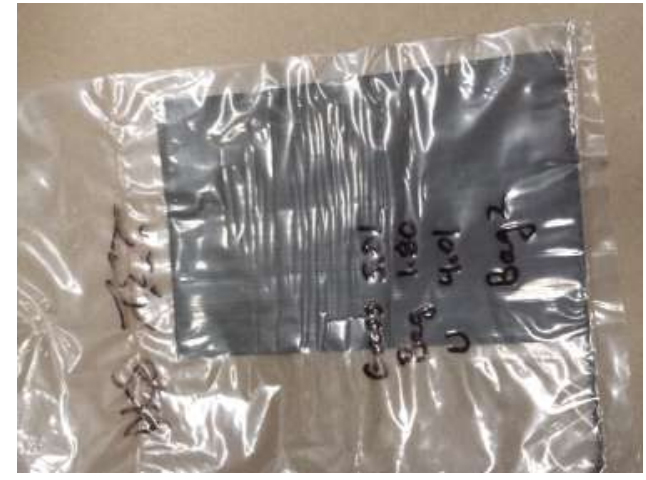

Fig. 1: 93.19\% enriched metallic uranium foil.

Based on these reported behaviors, it may be possible to use piezoelectric materials to detect lower energy radiation through the force applied on the piezoelectric materials by radiation. To test this assumption, an experimental set up to irradiate PZT plates with fission fragments was designed and evaluated for radiation safety and feasibility. Fission fragments were chosen as the initial experimental radiation source due to their high energy and large mass, which will results in large amounts of energy being deposited in a short distance. To create a source of fission fragments a metallic uranium foil, shown in Figure 1, enriched to 93.19\%, will be irradiated with a neutron source.

PZT was chosen as the first piezoelectric material to be tested since it is inexpensive and commonly available. A frame, shown in Figures 2 and 3 was then constructed to support the uranium foil, which is very fragile, and the PZT plates. The foil will be positioned between two sheets of mylar in order to provide contamination control and placed on top of an aluminum plate. A nylon plate was used for the 


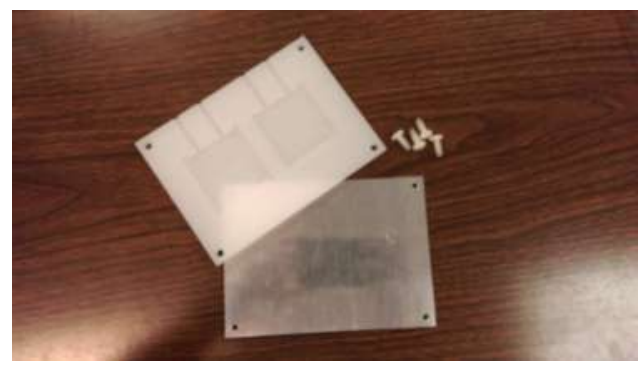

Fig. 2: Aluminum and nylon frame used to hold uranium foil and PZT plates.

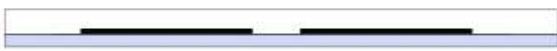

Fig. 3: Cross section view of the frame. PZT is indicated by black, Aluminum is indicated by gray and nylon is indicated by white.

other half of the frame. The nylon plate has two sets of wells and channels carved into it to hold two PZT plates and the wiring.

Two possible options of neutron source are available and have been evaluated for feasibility with the proposed experimental geometry: a 1 Curie plutonium-beryllium (PuBe) source and the thermal neutron beam-line at the Breazeale Reactor at Penn State. The PuBe source has a strength of $1.4 \mathrm{e} 6$ neutrons/second and, for the purpose of these evaluations, it was assumed to be an isotropic point source. The thermal neutron beam-line has a flux of $3.7 \mathrm{e} 7$ neutrons $/ \mathrm{cm}^{2 *}$ second and a 7 inch diameter [3]. It was assumed that the beam-line neutrons are mono-energetic with an energy of $.025 \mathrm{eV}$. Both neutron sources can be placed at various distances from the uranium foil. For the models discussed in this paper the neutron source was always placed $1 \mathrm{~cm}$ from the nylon plate.

\section{Calculation of Ranges of Fission frag- MENTS IN PZT}

The first step in determining the fission fragment exposure of the PZT plates in the geometry was to determine how far the fission fragments created in the uranium foil would be able to travel. This distance controls what percentage of the fission fragments are able to escape the foil and interact with the PZT plates. To find the range of the fission fragments, models using both Stopping Range of Ions in Matter (SRIM) and Monte Carlo N-Particle Transport 6 code (MCNP6) were evaluated [4], [5]. For these models it was assumed that the uranium foil was $100 \%$ uranium- 235 .

SRIM is an open source software that uses quantum mechanics to calculate ion transport data. For the SRIM range calculations, an infinitely thick plate of uranium-235 was modeled. This plate was bombarded with a variety of mono-energetic heavy ions, each run using a different heavy ion, and the resulting ranges were tabulated. For these models the heavy-ions were all given an energy of $77 \mathrm{MeV}$, since $77 \mathrm{MeV}$ is the average energy of a fission

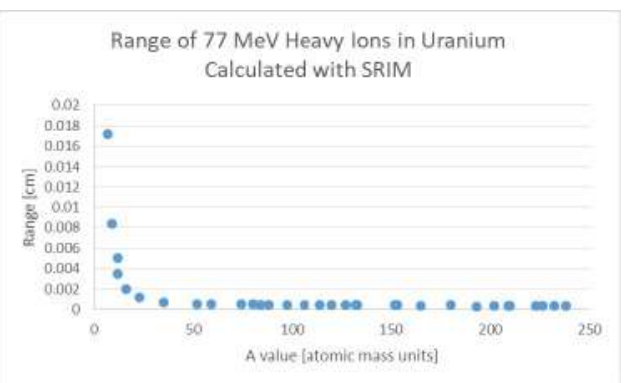

Fig. 4: Ranges for $77 \mathrm{MeV}$ heavy ions as calculated with SRIM.

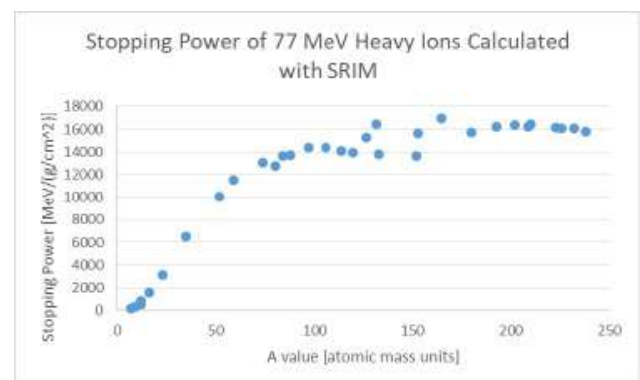

Fig. 5: Stopping powers for $77 \mathrm{MeV}$ heavy ions as calculated with SRIM.

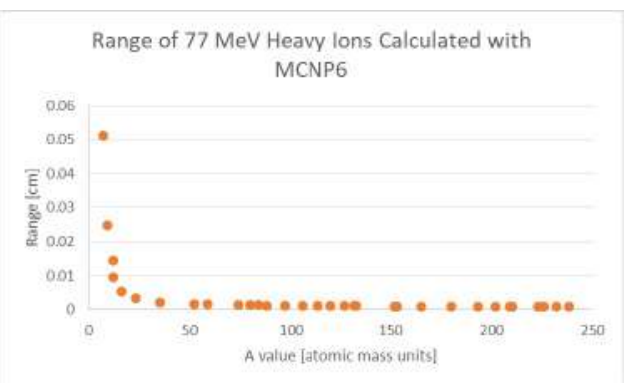

Fig. 6: Ranges for $77 \mathrm{MeV}$ heavy Ions as calculated with MCNP6.

fragment created by fissioning a uranium-235 nucleus. The stopping power, which describes the energy lost per unit path length of the radiation in a particular material, was also calculated with these SRIM models and tabulated. The plots of the resulting ranges and stopping power are shown in Figures 4 and 5.

For comparison the range and stopping power of the same selection of $77 \mathrm{MeV}$ heavy ions were then calculated using MCNP6. MCNP6 is a Monte Carlo method based radiation transport code. It uses a combination of the Janni proton range data tables and SPAR FORTRAN code with a density correction factor to calculate the geometry independent ranges and stopping powers for heavy ions in different target materials [6], [7]. The plots of the resulting ranges and stopping powers are shown in Figures 6 and 7.

The resulting ranges from both the SRIM and MCNP6 models were very similar for $77 \mathrm{MeV}$ ions in uranium-235 and both followed the same trend of the range rapidly decreasing initially, then trailing off as the atomic mass value of the ions increased. The lightest ion had a range of .51 


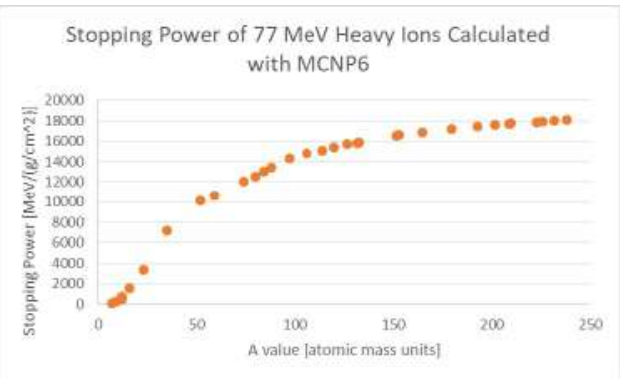

Fig. 7: Stopping powers for $77 \mathrm{MeV}$ heavy ions as calculated with MCNP6.

$\mathrm{mm}$ and the heaviest had a range of $3.8 \mu \mathrm{m}$, which indicates that a portion of the fission fragments born in the foil will be able to escape the foil and interact with the PZT plates. The ranges calculated by MCNP6 were in general lower than those calculated using SRIM. The stopping powers calculated using SRIM show artifacts that are likely due to interpolations for points where there is no data available. The MCNP6 stopping power results were smooth and were typically slightly lower than those calculated with SRIM.

\section{Source Selection: Fission Rate, fission Fragment Production, and Fission fragment Flux Calculations}

In order to chose which neutron source is the better option, the fission fragment flux that the PZT plates will be exposed to, was calculated. First, the fission rates were calculated using a model of the experimental geometry in MCNP6 and the given source strengths. For the PuBe source, the fission rate in the uranium foil for the given geometry was determined to be $7.4 \mathrm{e} 2$ fissions/second. For the thermal neutron beam-line, the fission rate was calculated to be 2.2e8 fissions / second in the uranium foil. To determine the fission fragment production rate the fission rate was doubled, since fissions typically produce two fission fragments. This resulted in fission fragment production rates of $1.5 \mathrm{e} 3$ fission fragments/second for the PuBe source and 4.4e 8 fission fragments/second for the thermal neutron beam-line.

Next, the average range of the average mass fission fragments was determined using SRIM, so that the volume of uranium foil contributing fission fragments to the fission fragment flux, which the PZT plates will interact with, could be determined. The average energy of a fission fragment from a uranium-235 fission was already determined to be 77 $\mathrm{MeV}$ and the average mass was calculated to be 117 atomic mass units. This gives a range of $4.69 \mathrm{e}-4 \mathrm{~cm}$. Since the PZT plates are $45 \mathrm{~mm}$ by $45 \mathrm{~mm}$, this gives a contributing foil volume of $.0095 \mathrm{~cm}^{3}$ for each of the PZT plates.

The fission rates were then normalized by volume. This resulted in a volumetric fission rate of $1.1 \mathrm{e} 3$ fissions $/$ second ${ }^{*} \mathrm{~cm}^{3}$ for the PuBe source and a volumetric fission rate of $3.24 \mathrm{e} 8$ fissions $/$ second ${ }^{*} \mathrm{~cm}^{3}$.

The fission fragment flux into each foil was then calculated assuming that each fission could only contribute one fission fragment, due to the directionality of the fission fragment trajectories. Using the volume normalized fission rates and the average range, the fission fragment

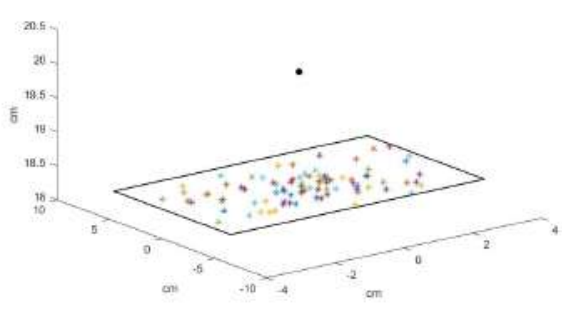

Fig. 8: Fission fragments born in the uranium foil. The PuBe source is indicated by the black dot.

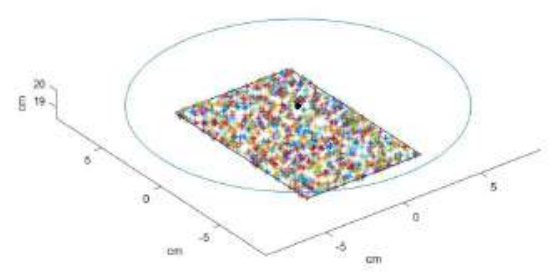

Fig. 9: Fission fragments born in the uranium foil. The thermal neutron beam-line is centered on the black dot and its perimeter is represented by the blue circle.

flux into each PZT plate was determined to be .511 fission fragments $/$ second ${ }^{*} \mathrm{~cm}^{2}$ for the PuBe source and 1.5e5 fission fragments/second ${ }^{*} \mathrm{~cm}^{2}$ for the thermal neutron beam-line.

To be able to understand the $2 \mathrm{D}$ distribution of the fissions and fission fragment production in the foil, MCNP6 Particle Track (PTRAC) outputs were used to create visualizations of the fission fragments being born in the foil. The PTRAC files were filtered and analyzed using Matlab scripts, in order to determine the location of the births of all the heavy ions in the foil. These were recorded and plotted, as seen in Figures 8 and 9, for both of the neutron sources. The PuBe source model in MCNP6 used 967,864,929 starting neutrons and the thermal neutron beam-line model used $1,248,130,865$ starting neutrons. It can be seen in these results that the PuBe source created in a clustering of fission in the center due to it being an isotropic point source, while the thermal neutron beam-line gave a relatively uniform distribution over the surface of the foil. The PTRAC file was then analyzed to create visualizations of the heavy ion particle tracks that were born in the foil and entered the PZT. These visualizations are shown in Figures 10 and 11. Once again the PuBe source results in a higher density of fission fragments towards the center while the thermal neutron beam-line results in an relatively even distribution over the foil area.

\section{PZT Sample Preparations}

For the first round of experiments using the proposed experimental geometry, PZT plates were purchased. In order to prepare them for the experiments, custom contacts were created and technique for wiring the samples was developed. The custom contacts were patterned to provide differing 


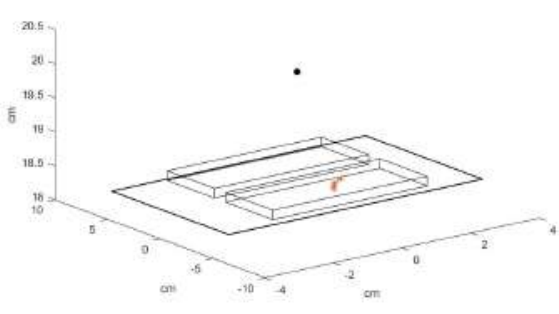

Fig. 10: Fission fragment tracks leaving the foil and entering the PZT plates. The PuBe source is centered on the black dot.

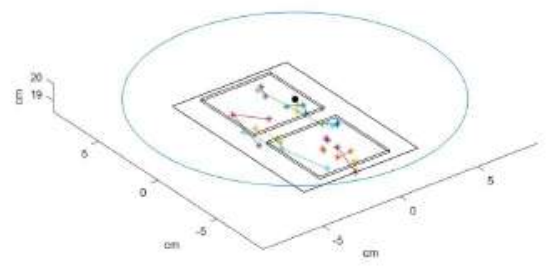

Fig. 11: Fission fragment tracks leaving the foil and entering the PZT plates. The thermal neutron beam-line is centered on the black dot and its perimeter is represented by the blue circle.

amounts of exposed PZT. This exposed PZT is important since it allows the fission fragments to deposit more energy directly into the PZT, instead of having to pass through the silver contact. A variety of comb shaped patterns were created with different comb tooth widths to give a range of electrode area coverages. Table 1 shows the various areas and comb widths for the contacts. For piezoelectric materials it is important to keep the material under its Curie temperature: the temperature threshold where the crystalline structure goes through a phase change and it looses its poling. To ensure the PZT plates remained under their Curie temperature of 320 degrees Celsius all preparations occurred at room temperature and required no heating.

For the contact preparations, the original fired-on silver electrodes were removed by polishing with $\mathrm{Al}_{2} \mathrm{O}_{3}$. A mask was then created with Kapton tape on the surface to protect the exposed PZT surface area when a new patterned silver contact was sputtered on. The PZT plate was covered on all exposed surfaces with Kapton tape. Then the mask was cut into the Kapton tape using a lazer cutter and the excess Kapton tape was removed. A masked PZT plate is shown in Figure 12. The sputtered silver was put on with a thickness of $100 \mathrm{~nm}$. An example of the final custom contacts are shown in Figure 13. The wiring for each PZT plate was attached to a ring terminal which was connected to the PZT plate using a silver conductive epoxy. A wired sample is shown in Figure 14.

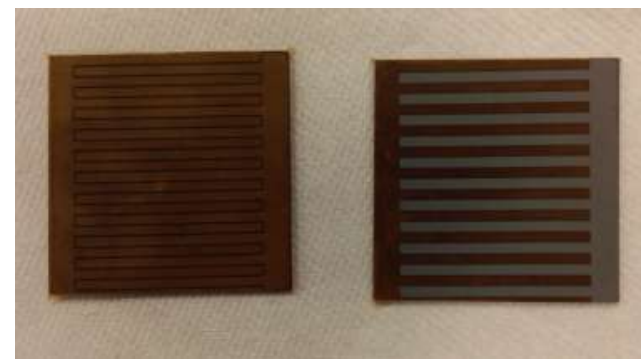

Fig. 12: Kapton tape masking on PZT plates after cutting on the left, and post removal of excess Kapton tape on the right.

TABLE 1: Custom electrode areas and comb teeth widths.

\begin{tabular}{cccc}
\hline $\begin{array}{c}\text { width of non- } \\
\text { electrode } \\
\text { bands [mm] }\end{array}$ & $\begin{array}{c}\text { width of } \\
\text { electrode } \\
\text { bands [mm] }\end{array}$ & $\begin{array}{c}\text { percent } \\
\text { area non- } \\
\text { electrode }\end{array}$ & $\begin{array}{c}\text { percent } \\
\text { area } \\
\text { electrode }\end{array}$ \\
\hline 2 & 2 & 49 & 51 \\
2 & 4 & 40 & 60 \\
2 & 8 & 30 & 70 \\
2 & 10 & 25 & 75 \\
2 & 2 & 51 & 49 \\
4 & 2 & 60 & 40 \\
8 & 2 & 70 & 30 \\
10 & 2 & 75 & 25 \\
\hline
\end{tabular}

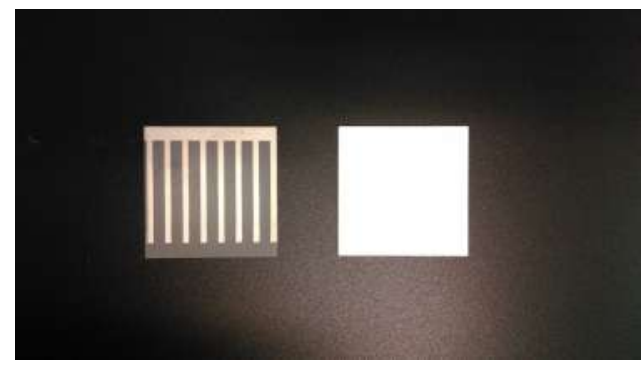

Fig. 13: PZT plate with solid commercial electrode on the right and a custom sputtered electrode on the left. The custom electrode coves $40 \%$ of the surface area of the face of the plate.

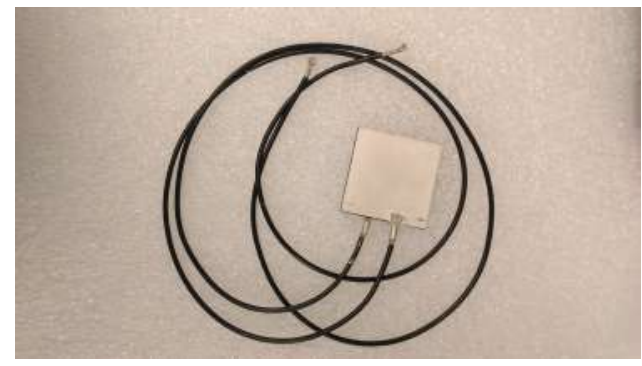

Fig. 14: PZT plate with commercial electrodes, epoxied ring terminals and wiring.

\section{Conclusions ANd Future Work}

For the proposed experimental geometry including the frame, uranium foil, PZT plates, and neutron source, the thermal neutron beam-line at the Breazeale Reactor will provide the larger fission fragment flux entering the PZT plates. This larger fission fragment flux will provide a greater force 
on the PZT plates, which will result in the production of a larger electrical signal. This fission fragment flux will be a maximum of $1.5 \mathrm{e} 5$ fission fragments $/$ second ${ }^{*} \mathrm{~cm}^{2}$.

The next step in this project will be to implement the experimental geometry evaluated in this paper. This will begin with PZT plates that have been prepared and will use a decreasing distance from the neutron source to control the fission fragment flux. In the future, other types of radiation, instead of fission fragments, will be tested including alpha particles and neutrons. Other piezoelectric materials will also be considered. PZT is very common but is also considered relatively resistant to radiation [8] therefore custommade, highly sensitive piezoelectric foils will also be tested.

\section{REFERENCES}

[1] A. Arnau and D. Soares, "Fundamentals of piezoelectricity," Piezoelectric Transducers and Applications, pp. 1-38, 2008.

[2] S. Takechi, T. Miyachi, M. Fujii, N. Hasebe, K. Mori, H. Shibata, T. Murakami, Y. Uchihori, and N. Okada, "Radiation detector based on piezoelectric lead zirconate titanate material," Nuclear Instruments \& Methods in Physics Research Section a-Accelerators Spectrometers Detectors and Associated Equipment, vol. 586, pp. 309-313, 2008.

[3] J. J. Duderstadt and L. J. Hamilton, "Nuclear Reactor Analysis," pp. 138-142, 1976.

[4] J. F. Ziegler, "SRIM-The Stopping and Range of Ions in Matter," 2013. [Online]. Available: www.srim.org

[5] "MCNP6-a general Monte Carlo N-Particle Transport Code, version 6.1," Los Alamos, New Mexico, 2013.

[6] J. F. Janni, "Energy loss, range, path length, time-of-flight, straggling, multiple scattering, and nuclear interaction probability." Atomic Data and Nuclear Data Tables, vol. 27, no. 2-3, pp. 147-339, 1982.

[7] T. W. Armstrong and K. C. Chandler, "Stopping powers and ranges for muons, charged pions, protons, and heavy ions," Nuclear Instruments and Methods, vol. 113, no. 2, pp. 313-314, 1973.

[8] A. Holmes-Siedle and L. Adams, The Handbook of Radiation Effects, 2nd ed. Oxford University Press, 2002. 